\title{
miR-222 induces apoptosis in human intervertebral disc nucleus pulposus cells by targeting Bcl-2
}

\author{
WEI WANG ${ }^{1}$, JIAN WANG ${ }^{2}$, JIAYI ZHANG ${ }^{2}$, WEI TAQ $^{3}$ and ZHENXING ZHANG ${ }^{4}$ \\ Departments of ${ }^{1}$ Rehabilitation, ${ }^{2}$ Orthopedics, ${ }^{3}$ General Surgery and ${ }^{4}$ Neurosurgery, \\ The First Affiliated Hospital of Jinzhou Medical University, Jinzhou, Liaoning 121000, P.R. China
}

Received March 11, 2019; Accepted August 6, 2019

DOI: $10.3892 / \mathrm{mmr} .2019 .10732$

\begin{abstract}
Intervertebral disc degeneration (IDD) is characterized by abnormal induction of apoptosis in intervertebral disc nucleus pulposus (NP) cells. Previous studies indicated that miR-222 was upregulated in patients with rheumatoid arthritis. However, the effects of miR-222 in IDD remain unclear. The present study aimed to demonstrate the role of miR-222 in NP cells. The levels of miR-222 in patients with IDD were measured by reverse transcription-quantitative PCR. Cell Counting Kit-8 and western blotting assays were used to detect cell proliferation and apoptosis-associated protein levels, respectively. In addition, luciferase reporter assays were performed to validate the predicted target genes of miR-222. miR-222 was significantly upregulated in patients with IDD. Overexpression of miR-222 inhibited cell proliferation and induced cell apoptosis. Moreover, overexpression of miR-222 resulted in an upregulation in the levels of Bax and cleaved caspase 3 , and a downregulation in the levels of $\mathrm{Bcl}-2$ in NP cells. The luciferase reporter assays demonstrated that $\mathrm{Bcl}-2$ is a target of miR-222. Furthermore, overexpression of miR-222 increased the levels of cytochrome c, apoptotic protease activating factor- 1 and cleaved caspase 9 in NP cells. Conversely, downregulation of miR-222 could promote the proliferation of NP cells. The present data demonstrated that miR-222 induced apoptosis in NP cells by directly targeting Bcl-2. Therefore, miR-222 may act as a potential therapeutic target for the treatment of IDD.
\end{abstract}

\section{Introduction}

Low back pain is a common and chronic disease (1). In total, $>80 \%$ of adults will suffer from back pain at a certain stage

Correspondence to: Dr Zhenxing Zhang, Department of Neurosurgery, The First Affiliated Hospital of Jinzhou Medical University, 2 Renmin Street, Section 5, Guta, Jinzhou, Liaoning 121000, P.R. China

E-mail: zhenxing_zhang666@126.com

Key words: microRNA-222, intervertebral disc degeneration, human intervertebral disc nucleus pulposus cells, Bcl-2, apoptosis in their lives (2). Intervertebral disc degeneration (IDD) is a common cause of lower back pain, and $20-33 \%$ of the patients with this disorder will not be able to work $(3,4)$. Moreover, IDD is also a prevalent musculoskeletal disorder, which may serve as a social and economic burden worldwide (5). At present, endoscopic lumbar discectomy is the common treatment method for IDD. However, this method may lead to recurrence of the disease following surgery $(6,7)$. At present, no effective preventative strategy and therapeutic method has been reported for treating IDD, due to the diversity and the unclear cause of low back pain. Therefore, it is important to identify the mechanism responsible for the development of IDD.

MicroRNAs (miRNAs) are endogenous short non-coding RNAs, which consist of 20 nucleotides (8). miRNAs play vital roles in the regulation of gene expression by controlling related physiological processes (9). Previous studies reported that miRNAs are involved in cell growth, proliferation and cell death in different diseases (10-12). It has been shown that some miRNAs exert an important role in the development of IDD, such as miR-194, miR-3150a and miR-125b-1 (13-15).

miR-222 acts as a tumor promoter in different cancer types, such as pancreatic and breast cancer $(16,17)$. miR-222 regulates the inflammatory response of macrophages in bacterial pneumonia (18). In addition, miR-222 regulates marrow niche-supported tumor cells, which promote survival of residual cells (19). A previous study indicated that the levels of miR-222 were upregulated in patients with rheumatoid arthritis (20). However, although various previous studies have been conducted on the biological functions of miR-222, the role of miR-222 in IDD remains unclear. In the present study, evidence is presented supporting the role of miR-222 in inducing the apoptosis of NP cells via Bcl-2 regulation.

\section{Materials and methods}

Patient samples. A total of 20 lumbar nucleus pulposus specimens were collected from patients with IDD between April 2017 and May 2018; the patients included 12 females and 8 males (ranging 43-65 years old) in the First Affiliated Hospital of the Jinzhou Medical University. Patients with diabetes mellitus, spondylolisthesis, serious systemic disease, ankylosing spondylitis, trauma history of the spine, or back surgery were excluded. In total, 20 matched healthy controls, recruited from public schools, were included in the 
present study. The present study was approved by the Ethics Committee of the First Affiliated Hospital of the Jinzhou Medical University, and informed consent was obtained from each participant prior to enrollment.

Cell culture and transfection. Human intervertebral disc nucleus pulposus (NP) cells were obtained from the Tongpai Biological Technology Co., Ltd. (http://www.shtpbio. com/tongpai-Products-3469763/). These cells are primary cells. NP cells were cultured in DMEM (Gibco; Thermo Fisher Scientific, Inc.) containing 10\% FBS (Gibco; Thermo Fisher Scientific, Inc.), $100 \mu \mathrm{g} / \mathrm{ml}$ streptomycin and $100 \mathrm{U} / \mathrm{ml}$ penicillin. The cells were incubated at $37^{\circ} \mathrm{C}$ in a humidified atmosphere with $5 \% \mathrm{CO}_{2}$.

The $10 \mathrm{nM}$ miR-222 mimics, $10 \mathrm{nM}$ miR-222 inhibitor and $10 \mathrm{nM}$ mimics negative control (NC) were obtained from Shanghai GenePharma Co., Ltd. Briefly, the cells ( $3 \times 10^{5}$ cells/well) were plated in 6 -well plates overnight. The sequence for miR-222 mimics was: 5'-AGCUACAUCUGG CUACUGGGU-3'. The sequence for miR-222 inhibitor was: 5'-ACCCAGUAGCCAGAUGUAGCU-3'. The sequence for NC was 5'-UUUGUACUACACAAAAGUACUG-3'. Subsequently, miR-222 mimics or miR-222 inhibitor were transfected into the cells using Lipofectamine ${ }^{\circledR} 2000$ (Thermo Fisher Scientific, Inc.), according to the manufacturer's protocol. The transfection efficiency of miR-222 mimics in NP cells was assessed using reverse transcription-quantitative PCR (RT-qPCR) following $72 \mathrm{~h}$ of growth after cell transfection.

$R T-q P C R$. Total RNA extraction from the lumbar nucleus pulposus specimens and cells was performed using an RNA extraction kit (Total RNA Purification kit; Sigma Aldrich; Merck KGaA). Total RNA was reverse transcribed into cDNA using the TaqMan ${ }^{\circledR}$ MicroRNA RT kit (Applied Biosystems; Thermo Fisher Scientific, Inc.) according to the manufacturer's protocol. The RT reaction conditions were as follows: $37^{\circ} \mathrm{C}$ for $60 \mathrm{~min}$, followed by $85^{\circ} \mathrm{C}$ for $5 \mathrm{~min}$ and $4^{\circ} \mathrm{C}$. The cDNA samples were subjected to real-time PCR using a SYBR Green One-Step RT-qPCR kit (Thermo Fisher Scientific, Inc.) on an ABI 7900HT instrument (ABI; Thermo Fisher Scientific, Inc.). The qRCR reaction conditions were as follows: $95^{\circ} \mathrm{C}$ for $3 \mathrm{~min}$, followed by 40 cycles at $95^{\circ} \mathrm{C}$ for $10 \mathrm{sec}, 58^{\circ} \mathrm{C}$ for $30 \mathrm{sec}$ and $72^{\circ} \mathrm{C}$ for $30 \mathrm{sec}$. The primer sequences for miR-222 were as follows: Forward primer: 5'-AGCUACAUCUGGCUA CUGGGU-3' and reverse primer: 5'-CCAGUAGCCAGAUGU AGCUUU-3'. The primer sequences for U6 were as follows: Forward primer: 5'-CTCGCTTCGGCAGCACAT-3'; reverse primer: 5'-AACGCTTCACGAATTTGCGT-3'. All samples were tested in triplicate. The relative expression was measured using the $2^{-\Delta \Delta \mathrm{Cq}}$ method (21). Gene expression results were normalized by the internal control U6.

Cell Counting Kit-8 assay. Cell viability was assessed using CCK-8 (Beyotime Institute of Biotechnology). NP cells ( $2 \times 10^{4}$ cells/well) were seeded into 96 -well plates overnight. Subsequently, miR-222 mimics or miR-222 inhibitor were added into each well for $0,24,48$ and $72 \mathrm{~h}$. A total of $10 \mu \mathrm{l}$ CCK- 8 reagent was added into each well for $2 \mathrm{~h}$. The absorbance of each well was determined at $450 \mathrm{~nm}$ with a microplate reader (Molecular Devices, LLC).
Immunofluorescence assay. NP cells $\left(1 \times 10^{5}\right.$ cells/well) were plated into 24-well plates overnight. Subsequently, miR-222 mimics, miR-222 inhibitor or mimics control were added into each well. The cells were fixed in $4 \%$ paraformaldehyde for $20 \mathrm{~min}$ at room temperature, and in ice-cold $100 \%$ methanol for an additional $20 \mathrm{~min}$ at $-20^{\circ} \mathrm{C}$. After $30 \mathrm{~min}$ of blocking with $3 \%$ BSA in TBST at room temperature, the cells were incubated with the following antibodies: Anti-proliferation marker protein Ki-67 (Ki67; Abcam; cat. no. ab15580; 1:1,000), anti-Bcl-2 (Abcam; cat. no. ab32124; 1:1,000) and DAPI antibody (cat. no. ab104139; $1: 1,000$ ) at $4^{\circ} \mathrm{C}$ overnight. The following morning, horseradish peroxidase-conjugated Goat anti-rabbit IgG secondary antibodies (Abcam; cat. no. ab150081; $1: 5,000)$ were added for $1 \mathrm{~h}$ to the cells at room temperature. The cells were observed with a Leica confocal microscopy (Leica; magnification, $\mathrm{x} 400$ ) and the images were analyzed using the Leica LAS-X software.

Flow cytometry analysis of cell apoptosis. NP cells $\left(5 \times 10^{5}\right.$ cells/well) were seeded into 6 -well plates overnight. miR-222 mimics, or mimics control were added into each well for $72 \mathrm{~h}$. The cells were collected, washed 3 times with cold PBS and resuspended in 1X binding buffer. The cells were stained with dual-staining Annexin V-FITC-propidium iodide (PI; Thermo Fisher Scientific, Inc.) for $20 \mathrm{~min}$ at room temperature according to the manufacturer's protocol. The results were analyzed using a FACS Calibur flow cytometer (BD Biosciences) on a BD FACSCalibur system (FACScan, BD Biosciences).

Western blot analysis. The proteins were extracted from the cells using RIPA lysis buffer (Thermo Fisher Scientific, Inc.) and their concentration levels were quantified using a bicinchoninic acid protein assay kit (Beyotime Institute of Biotechnology). The proteins (40 $\mu \mathrm{g}$ per lane) were separated by SDS-PAGE on $10 \%$ gels, and were transferred onto $0.45 \mu \mathrm{m}$ PVDF membranes (Thermo Fisher Scientific, Inc.). Following blocking with $5 \%$ non-fat milk at room temperature for $1 \mathrm{~h}$, the membranes were incubated with primary antibodies overnight at $4^{\circ} \mathrm{C}$. The following primary antibodies were used: Anti-Bax (Abcam; cat. no. ab32503; 1:1,000), anti-Bcl-2 (Abcam; cat. no. ab32124; 1:1,000), anti-cleaved caspase 3 (Abcam; cat. no. ab2302; 1:1,000), anti-cytochrome c (Cyto C; Abcam; cat. no. ab13575; 1:1,000), anti-apoptotic protease activating factor-1 (Apaf 1; Abcam; cat. no. ab2001; 1:1,000), anti-cleaved caspase 9 (Abcam; cat. no. ab2324; $1: 1,000)$ and anti- $\beta$-actin (Abcam; cat. no. ab8227; 1:1,000). Subsequently, the membranes were incubated with horseradish peroxidase-conjugated secondary antibody (Abcam; cat. no. ab150077; 1:200) for $1 \mathrm{~h}$ at room temperature. Finally, an image of the protein band was detected using ECL reagent (Santa Cruz Biotechnology, Inc.). Image-Pro Plus software (version 7, Media Cybernetics, Inc.) was used for the targets that were normalized to $\beta$-actin.

Luciferase reporter assay. The potential target genes of miR-222 were predicted using miRanda (http://www.microrna. org), miRDB (http://www.mirdb.org/) and TargetScan (http://www.targetscan.org/). The target gene sequence and the mutation gene sequence were synthesized by Shanghai 
A

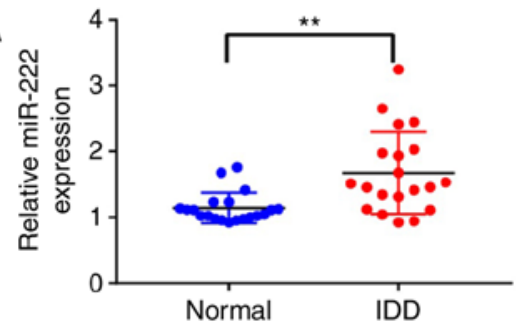

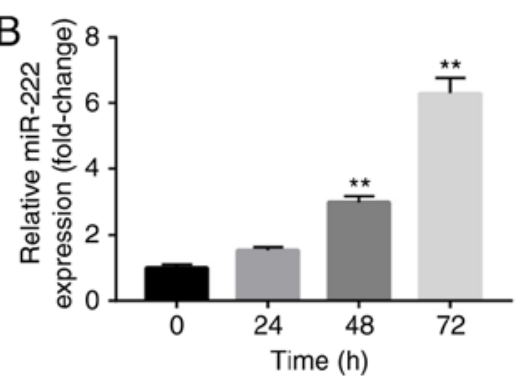

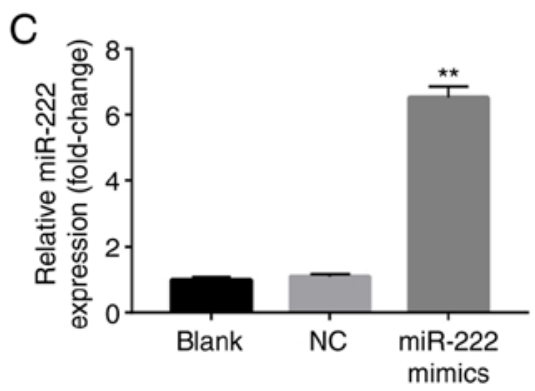

Figure 1. Increased miR-222 expression levels in IDD tissues and NP cells. (A) Relative expression levels of miR-222 in IDD and normal disc tissues were examined by reverse transcription-quantitative PCR. $n=20 .{ }^{* *} \mathrm{P}<0.01$. (B) Relative expression levels of miR-222 in NP cells following transfection with miR-222 mimics for $0,24,48$ and $72 \mathrm{~h}$. ${ }^{* *} \mathrm{P}<0.01$ vs. the $0 \mathrm{~h}$ group. (C) Relative expression levels of miR-222 in NP cells following transfection with the NC and miR-222 mimics for $72 \mathrm{~h} .{ }^{* *} \mathrm{P}<0.01$ vs. the NC group. miR, microRNA; IDD, intervertebral disc degeneration; NP, nucleus pulposus; NC, negative control.
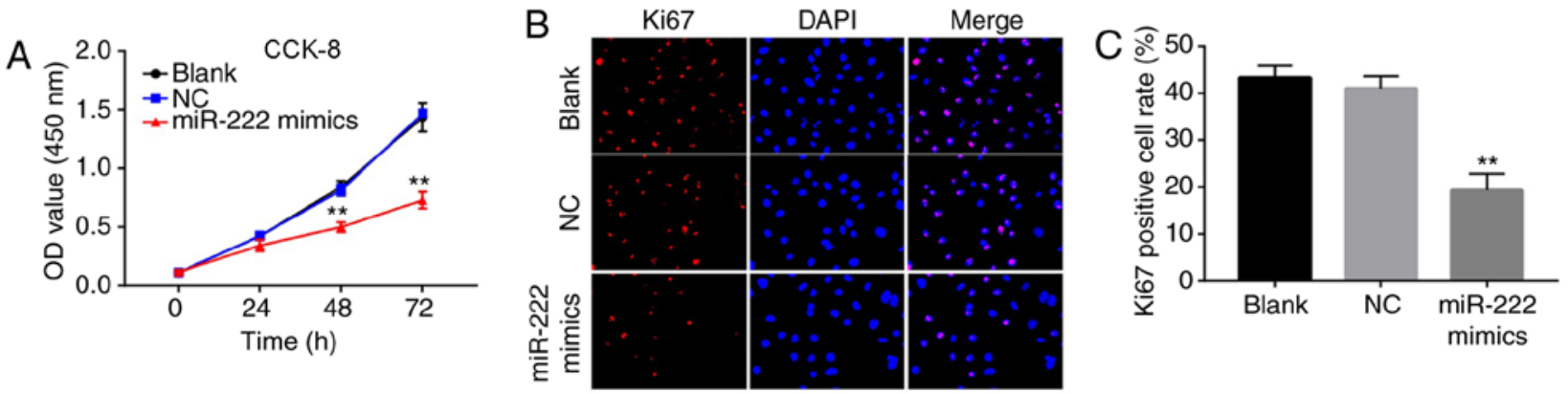

Figure 2. miR-222 overexpression inhibits proliferation of NP cells. (A) Cell viability of NP cells following transfection with NC and miR-222 mimics was determined by a CCK-8 assay at 0,24, 48 and $72 \mathrm{~h}$. Relative fluorescence expression levels were observed by (B) Ki67 and DAPI staining (magnification, $\mathrm{x} 400$ ), and (C) subsequent analysis. ${ }^{* *} \mathrm{P}<0.01$ vs. the NC group. miR, microRNA; NP, nucleus pulposus; CCK-8, Cell Counting Kit-8; Ki67, proliferation marker protein $\mathrm{Ki}-67$; NC, negative control; OD, optical density.

GenePharma Co., Ltd. The 3'-untranslated region (3'-UTR) of $B c l-2$ was amplified from genomic DNA and inserted into the psiCHECK-2 vector (Promega Corporation) using the XhoI and NotI sites. NP cells were co-transfected with psiCHECK2-Bcl-2-wild-type + miR-222 mimics, and psiCHECK2-Bcl-2-mutated type + miR-222 mimics using Lipofectamine ${ }^{\circledR} 2000$ (Thermo Fisher Scientific, Inc.). The cells were incubated in $5 \% \mathrm{CO}_{2}$ at $37^{\circ} \mathrm{C}$ for $48 \mathrm{~h}$. The dual luciferase reporter assay system (Promega Corporation) was used to measure luciferase activity. The firefly luciferase activity was normalized, according to Renilla luciferase activity.

Statistical analysis. Each sample was assessed for at least three independent determinations. Data are presented as mean \pm standard error. Graphs were generated using GraphPad Prism software (version 7.0, GraphPad Software, Inc.). The comparison between the two groups was analyzed by the Student's t-test. The comparisons among multiple groups were performed with one-way ANOVA followed by the Dunnett's test. $\mathrm{P}<0.05$ was considered to indicate a statistically significant difference.

\section{Results}

miR-222 expression levels are increased in IDD tissues and $N P$ cells. To investigate the role of miR-222 in the development of IDD, RT-qPCR was used to detect the levels of miR-222 in IDD and normal disc tissues. A total of 20 human IDD tissues were used with corresponding control samples. The mean levels of miR-222 were significantly increased compared with the normal group (Fig. 1A). In addition, RT-qPCR was used to detect miR-222 levels in NP cells, following transfection with miR-222 mimics for $0,24,48$ or $72 \mathrm{~h}$. The expression levels of miR-222 in NP cells were significantly increased following transfection with miR-222 mimics for 48 and $72 \mathrm{~h}$ (Fig. 1B). miR-222 mimics were further used in the present study to successfully increase the levels of miR-222 in NP cells. The levels of miR-222 were significantly upregulated in NP cells following transfection with miR-222 mimics for $72 \mathrm{~h}$ (Fig. 1C). These results indicated that the levels of miR-222 were increased in IDD tissues and NP cells.

miR-222 overexpression inhibits proliferation of NP cells. To study the effects of miR-222 on NP cells, a CCK- 8 assay was used to detect cell viability. Overexpression of miR-222 inhibited cell proliferation (Fig. 2A). Similarly, the results of the immunofluorescence assay demonstrated that the overexpression of miR-222 significantly decreased the number of Ki67 positive cells (Fig. 2B and C). The data suggested that miR-222 overexpression inhibited proliferation of NP cells.

miR-222 overexpression induces apoptosis of NP cells. To further determine whether miR-222 was responsible for the induction of apoptosis in NP cells, flow cytometry was employed to analyze the extent of apoptosis. The cell apoptotic rate was markedly increased in the miR-222 mimics group 
A

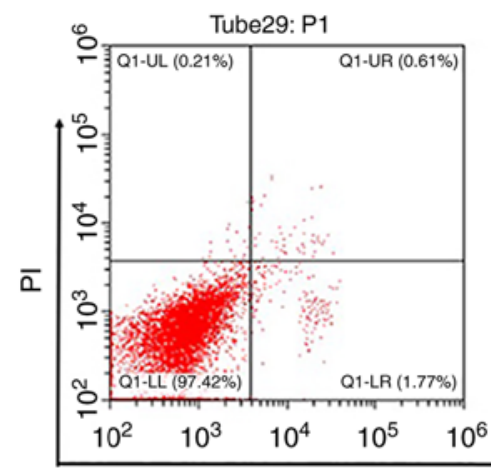

NC

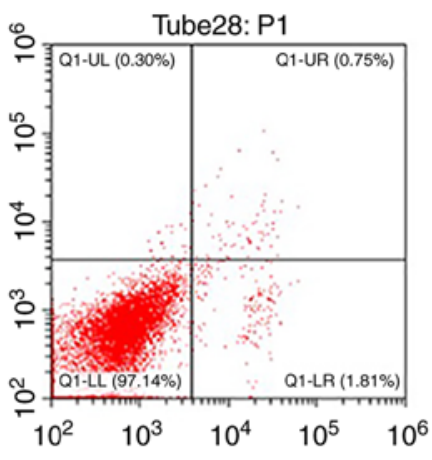

miR-222 mimics

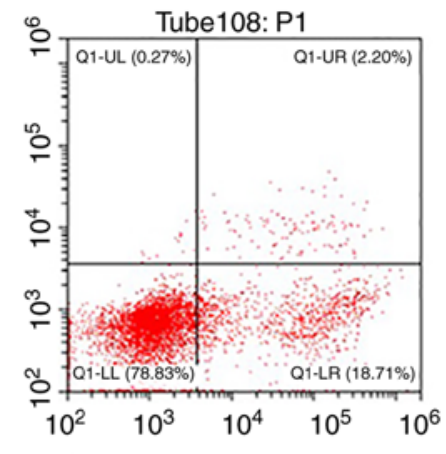

Annexin V

B

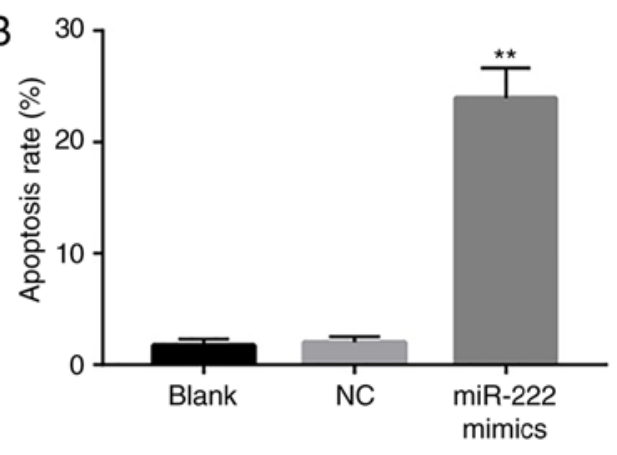

C

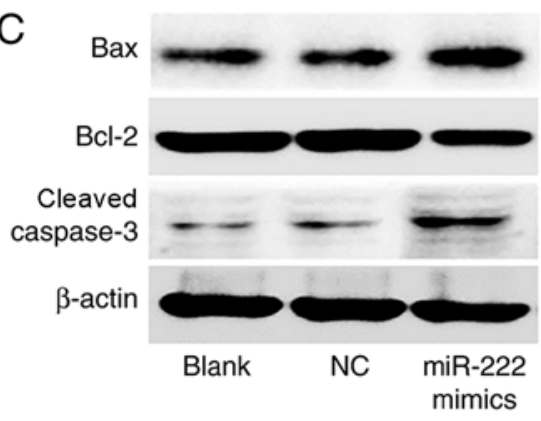

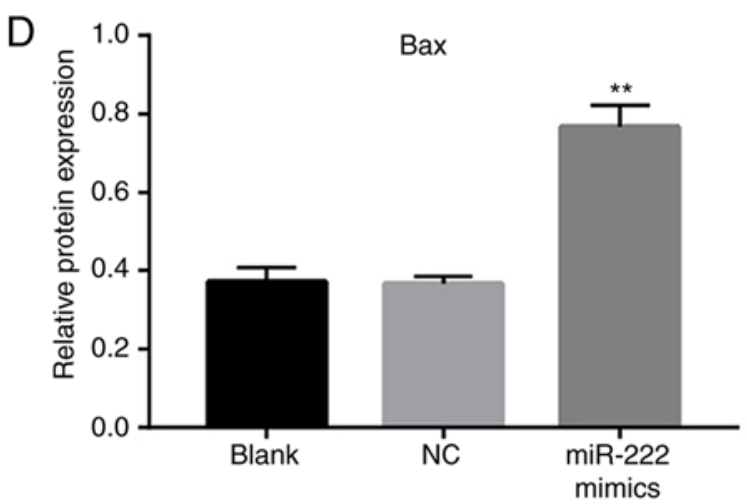
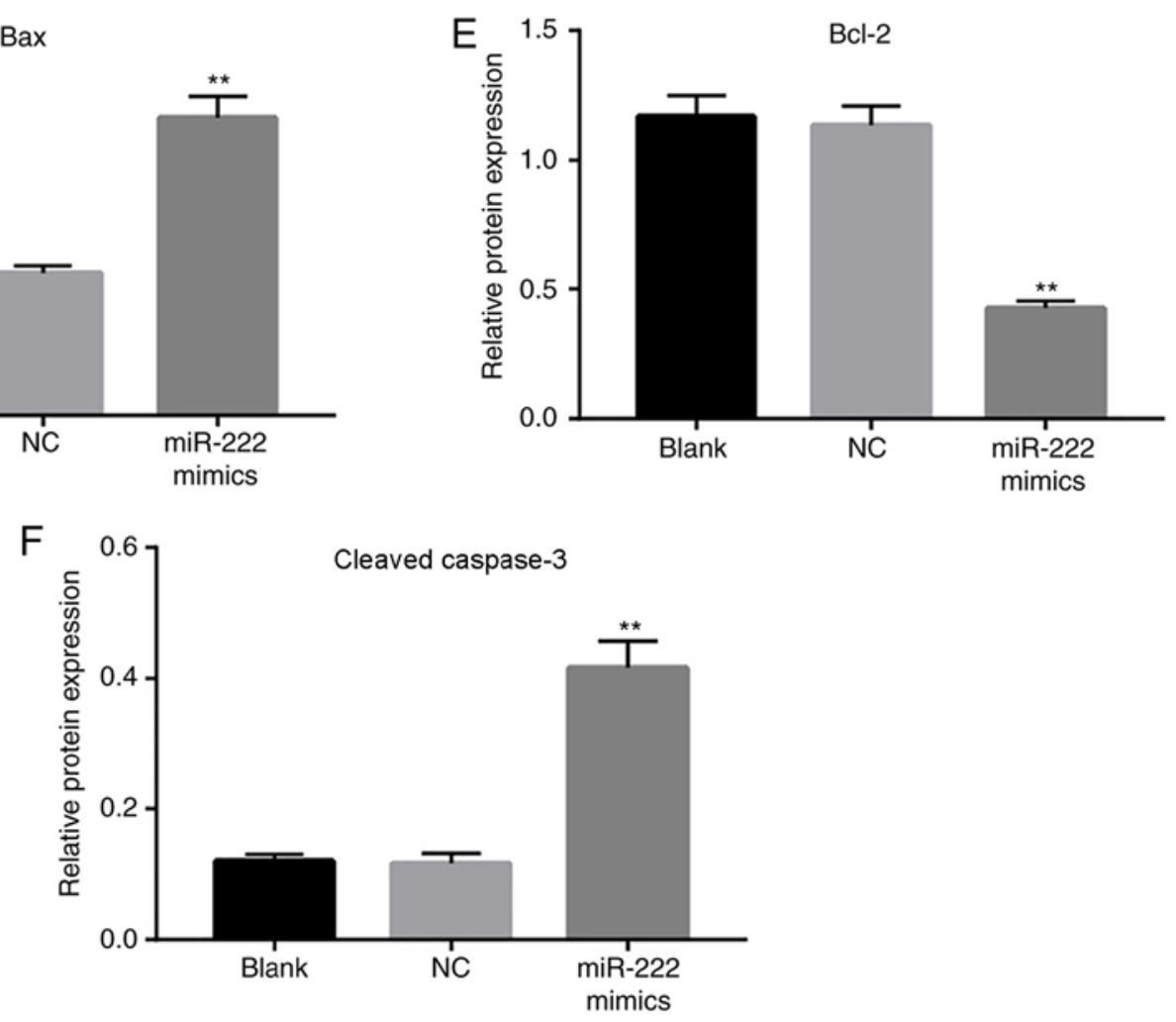

Figure 3. miR-222 overexpression induces apoptosis of NP cells. (A and B) Induction of apoptosis of NP cells following transfection with NC and miR-222 mimics. (C) Protein expression levels of Bax, Bcl-2 and cleaved caspase 3 in NP cells following transfection with NC and miR-222 mimics. Relative expression levels of (D) Bax, (E) Bcl-2 and (F) cleaved caspase 3 were quantified by normalizing them to the levels of $\beta$-actin. ${ }^{* *} \mathrm{P}<0.01$ vs. the NC group. miR, microRNA; $\mathrm{NP}$, nucleus pulposus; NC, negative control; PI, propidium iodide.

compared with the NC group (Fig. 3A and B). In addition, the expression levels of the apoptotic proteins Bax and cleaved caspase 3 were significantly increased, while the level of
Bcl-2 was reduced in the miR-222 mimics group (Fig. 3C-F). These data s that miR-222 overexpression induced apoptosis of NP cells. 


\section{A}

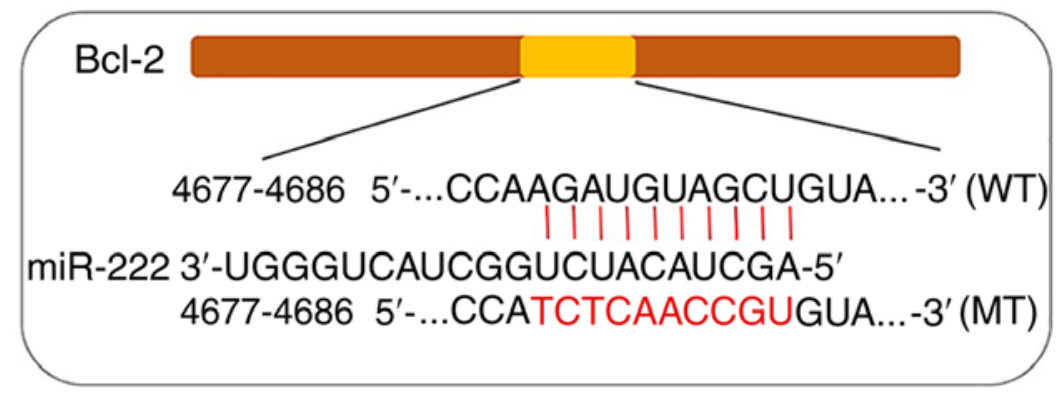

B
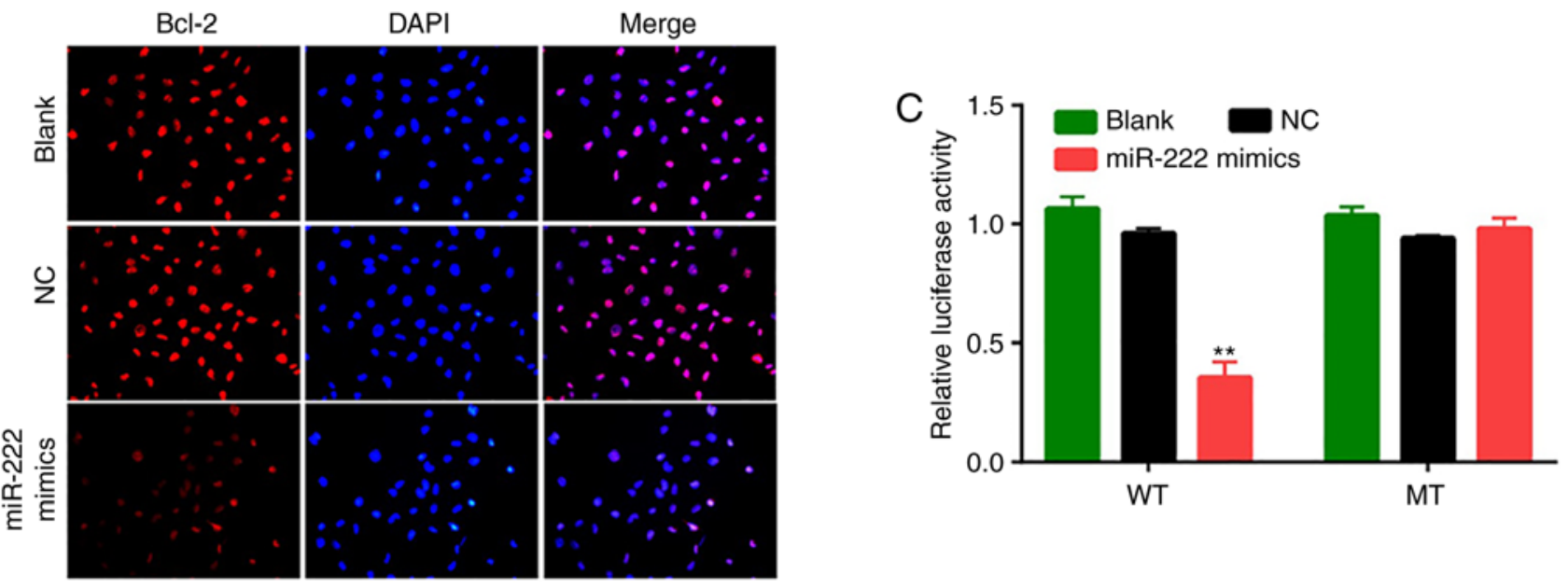

\section{Cyto C \\ Apaf 1 \\ Cleaved caspase-9 \\ $\beta$-actin}

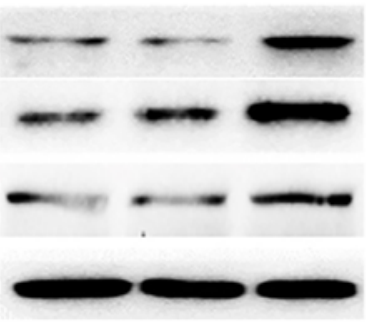

Blank NC miR-222

mimics

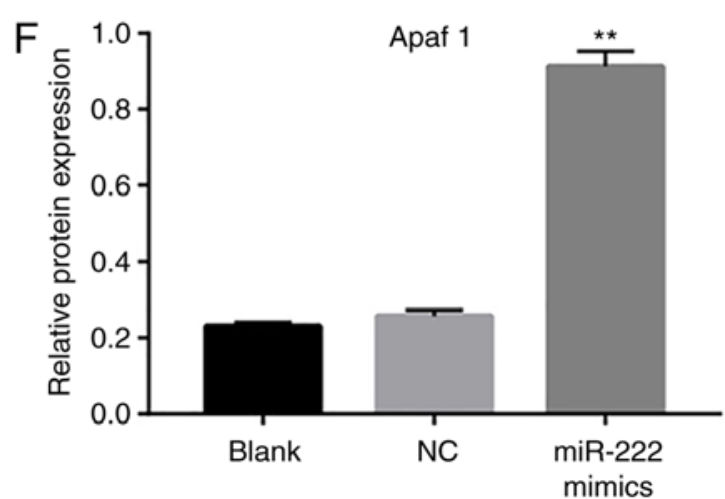

E

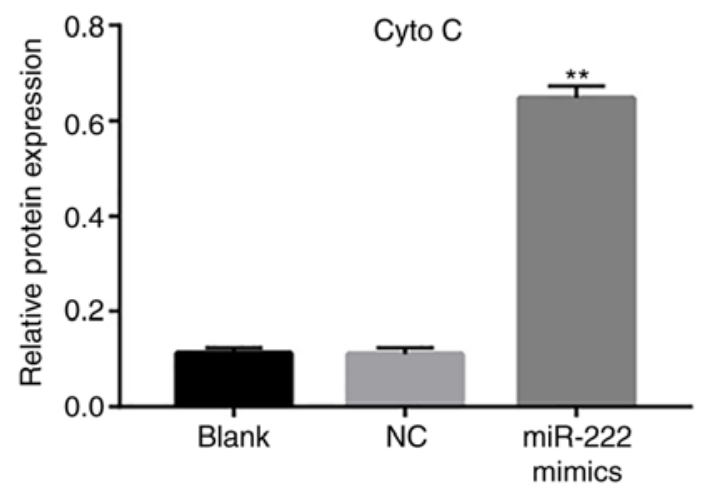

$\mathrm{G}$

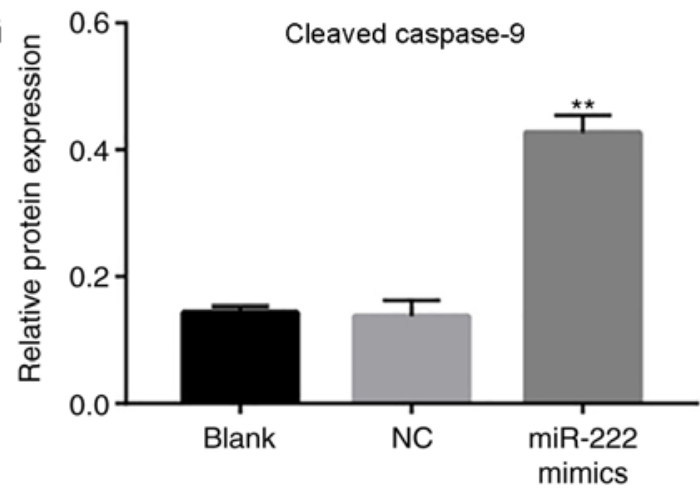

Figure 4. Bcl-2 is the target gene of miR-222. (A) Sequence alignment of human miR-222 with $B c l-2$. (B) NP cells were transfected with NC and miR-222 mimics, and the relative fluorescence expression levels were observed by Bcl-2 detection and DAPI staining (magnification, $\mathrm{x} 400$ ). (C) Luciferase reporter assays were used to detect the luciferase activity. The cells in the WT group were treated with psiCHECK2-Bcl-2-WT+miR-NC and psiCHECK-2-Bcl-2-WT+miR-222 mimics separately. The cells in the MT group were treated with psiCHECK-2-Bcl-2-MT+miR-NC and psiCHECK2-Bcl-2-MT+miR-222 mimics separately. Both firefly and Renilla luciferase activities were measured in the same sample. The firefly luciferase activity was normalized, according to Renilla luciferase activity. (D) Protein expression levels of Cyto C, Apaf 1 and cleaved caspase 9 detected in the intervertebral disc NP cells following transfection with NC and miR-222 mimics. Relative expression levels of (E) Cyto C, (F) Apaf 1 and (G) cleaved caspase 9 were quantified by normalizing them to the levels of $\beta$-actin. ${ }^{* *} \mathrm{P}<0.01$ vs. the NC group. miR, microRNA; NP, nucleus pulposus; NC, negative control; WT, wild-type; MT, mutant type; Cyto C, cytochrome C; Apaf 1, apoptotic protease activating factor-1. 

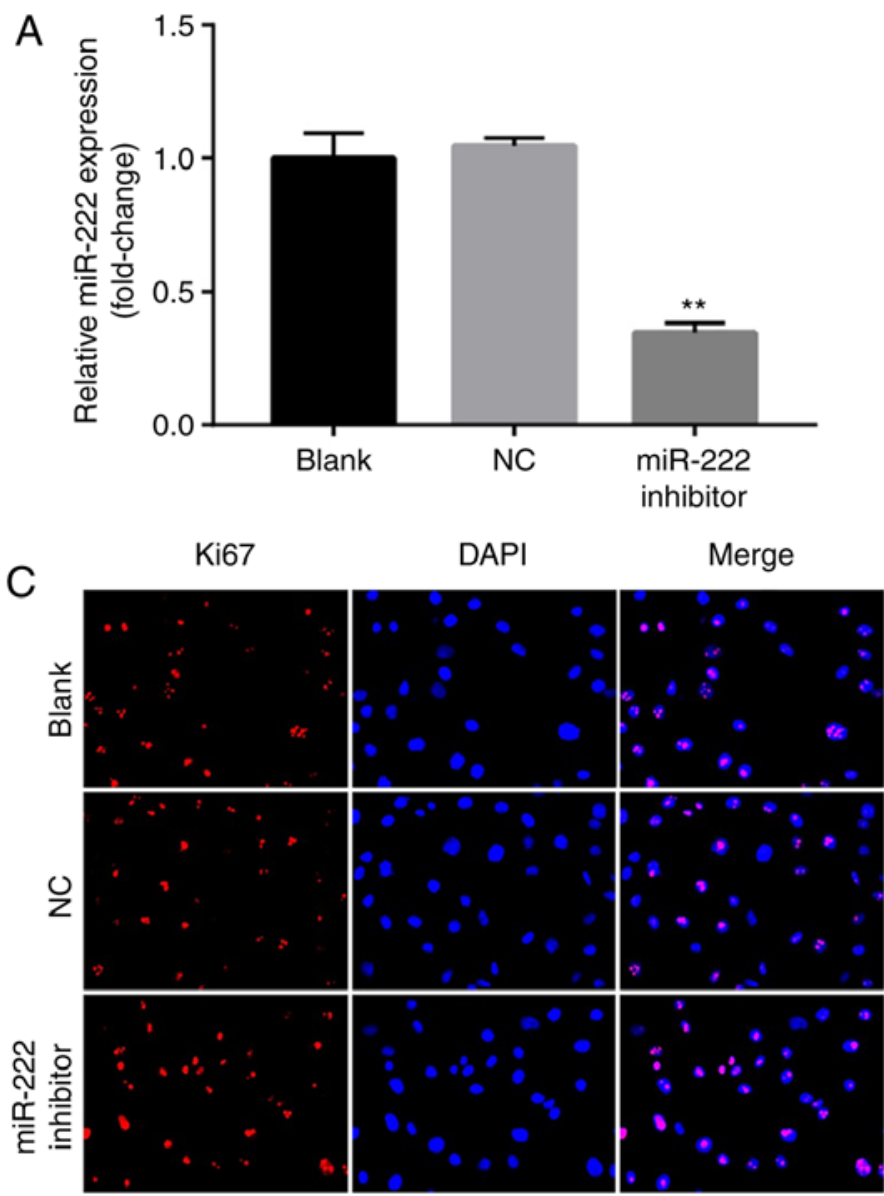
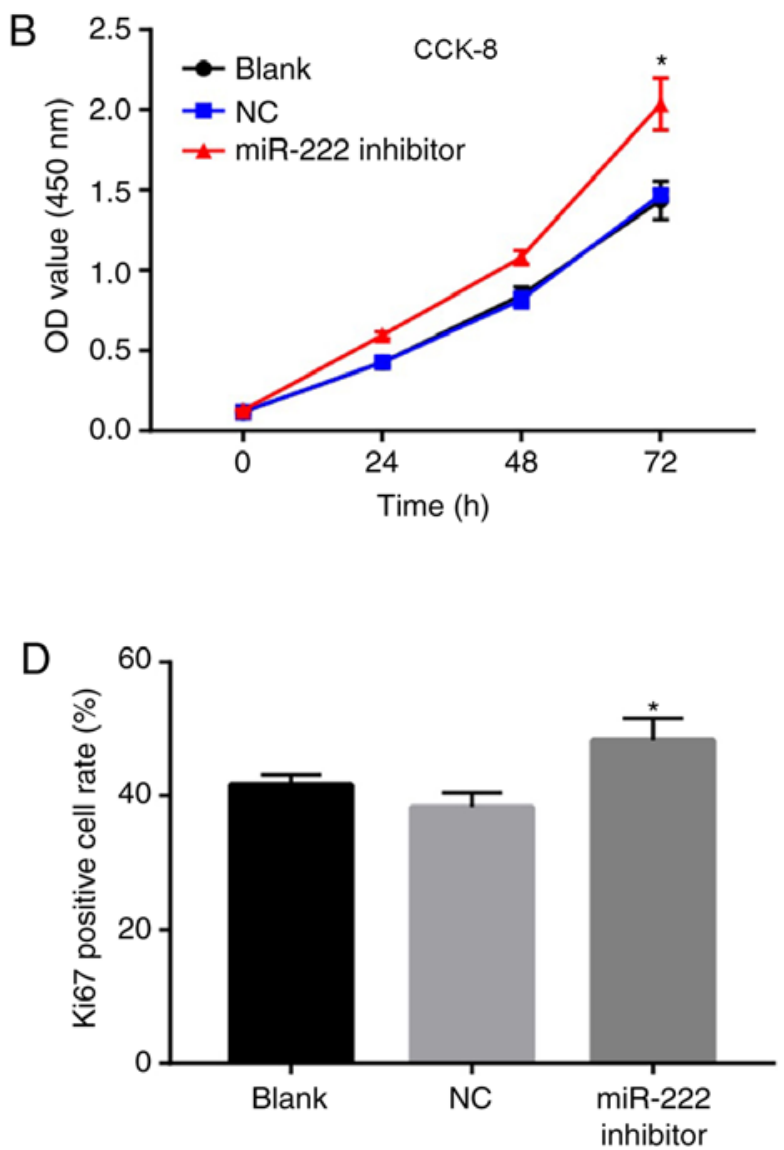

Figure 5. Downregulation of miR-222 promotes proliferation of NP cells. (A) Relative expression of miR-222 in NP cells following transfection with NC and miR-222 inhibitor for $72 \mathrm{~h}$. (B) Cell viability of NP cells following transfection with NC and miR-222 inhibitor was determined by a CCK-8 assay at 0, 24, 48 and $72 \mathrm{~h}$. Relative fluorescence expression levels were observed by (C) Ki67 detection and DAPI staining (magnification, $\mathrm{x} 400$ ), and (D) subsequent analysis. ${ }^{*} \mathrm{P}<0.05,{ }^{* * *} \mathrm{P}<0.01$ vs. the $\mathrm{NC}$ group. miR, microRNA; NP, nucleus pulposus; NC, negative control; CCK-8, Cell Counting Kit-8; Ki67, proliferation marker protein Ki-67; OD, optical density.

$B c l-2$ is a target gene of $m i R-222$. As predicted by miRanda, miRDB and TargetScan, there was complementary binding between miR-222 and $B c l-2$. The miR-222 sequence exhibited a binding site for the 3'-UTR of wild-type Bcl-2 (Fig. 4A). To further confirm the functional interaction between miR-222 and $B c l-2$, immunofluorescence and luciferase reporter assays were used to verify the binding of miR-222 to its target. The expression levels of $\mathrm{Bcl}-2$ were markedly decreased in the miR-222 mimics group compared with the NC group (Fig. 4B). In addition, overexpression of miR-222 markedly decreased the luciferase activity of the reporter gene with regard to the wild-type genotype, whereas the transfection of the cells with miR-222 mimics exhibited no significant effect on the mutant genotype, indicating that miR-222 directly targeted the 3'-UTR of Bcl-2 (Fig. 4C). In addition, the expression levels of the mitochondrial apoptotic pathway-associated proteins, Cyto C, Apaf 1 and cleaved caspase 9, were significantly increased in the miR-222 mimics group compared with the NC group. These data suggested that miR-222 induced cell apoptosis by targeting $\mathrm{Bcl}-2$ in NP cells.

Downregulation of miR-222 promotes proliferation of NP cells. The data derived from the previous experiments demonstrated that miR-222 overexpression could inhibit proliferation of NP cells. To further investigate the biological roles of miR-222 on NP cells, the cells were transfected with an miR-222 inhibitor. The levels of miR-222 were significantly downregulated in NP cells following transfection of the miR-222 inhibitor (Fig. 5A). In addition, downregulation of miR-222 promoted cell proliferation (Fig. 5B). Similarly, the results of the immunofluorescence assay suggested that downregulation of miR-222 increased Ki67 positive cells compared with the $\mathrm{NC}$ group (Fig. 5C and D). The present data suggested that downregulation of miR-222 promoted proliferation of NP cells.

\section{Discussion}

Despite the recent advances of experimental medicine, the therapeutic applications for IDD are still limited. Therefore, new therapeutic strategies are required for the treatment of IDD. In the present study, it was demonstrated that miR-222 levels were upregulated in IDD tissues. In addition, upregulation of miR-222 could inhibit proliferation and induce apoptosis of NP cells. Abo ElAtta et al (20) suggested that the expression levels of miR-222 were increased in patients with rheumatoid arthritis. In addition, Nie and Tian (22) indicated that miR-222 was upregulated in patients with uterine cancer. 
Therefore, these results indicated that miR-222 may play a role in inhibiting cell proliferation.

Apoptosis is a physiological process, which can remove harmful or seriously injured cells and organelles (23). A previous study indicated that the inhibition of apoptosis in NP cells could retard disc degeneration (24). A decrease in the number of NP cells was associated with the development of IDD, and inhibition of cell apoptosis was used as a strategy to reverse IDD (25). In the present study, the data demonstrated that miR-222 induced apoptosis in NP cells by reducing the levels of Bcl-2, and increasing the levels of Bax and cleaved caspase 3. Zhang et al (26) further demonstrated that miR-222 induced apoptosis in glioma cells by decreasing the levels of Bcl-2 and increasing the levels of Bax. These data suggested that miR-222 could induce apoptosis in NP cells.

In the present study, a novel mechanism of the miR-222-associated therapeutic action for the treatment of IDD was proposed. The present results identified that $\mathrm{Bcl}-2$ is a target gene of miR-222 in NP cells, as determined by a luciferase reporter assay. $\mathrm{Bcl}-2$ is an anti-apoptotic protein, which belongs to the Bcl-2 family of proteins, and is considered a classical biomarker of the mitochondrial pathway of apoptosis (27). In apoptosis, external stimuli, such as cytotoxic agents, could result in mitochondrial dysfunction, and then result in the release of pro-apoptotic protein Cyt c and activation of caspases to induce apoptosis (28). Previous studies demonstrated that the dissociation of the Bcl-2/Bax complex can lead to the release of Cyto C $(29,30)$. The proteins Cyto C, Apaf- 1 and caspase- 9 form the apoptosome, which increases the levels of cleaved caspase- 9 and leads to induction of apoptosis (30). In the present study, it was demonstrated that overexpression of miR-222 increased the levels of Cyto C, Apaf 1 and caspase 9 by targeting Bcl-2. The present data suggested that miR-222 induced apoptosis of NP cells via the mitochondrial-dependent apoptotic pathway. A decrease in the levels of Bcl-2 and a concomitant increase in the levels of cleaved caspase 3, cleaved caspase 9 and Bax were detected in NP cells, which was consistent with the data reported in a previous study (31). In addition, it is important to note that downregulation of miR-222 promoted proliferation of NP cells. The present findings demonstrated that overexpression of miR-222 induced apoptosis of NP cells via the mitochondrial-mediated apoptotic pathway. However, further studies are needed to investigate the role of miR-222 in animal model of intervertebral disc nucleus pulposus.

In conclusion, the present study demonstrated that miR-222 induces apoptosis in human NP cells by directly targeting Bcl-2. In addition, miR-222 may play a role in inhibiting cell proliferation and inducing cell apoptosis in human NP cells. Therefore, miR-222 may act as a potential therapeutic target for the treatment of IDD.

\section{Acknowledgements}

Not applicable.

\section{Funding}

The present study was funded by The Department of Science and Technology of the Liaoning Preparation of Animal Models and Animal Experimental Research for Major Diseases (grant no. 2012408002).

\section{Availability of data and materials}

The datasets used and/or analyzed during the present study are available from the corresponding author on reasonable request.

\section{Authors' contributions}

WW and JW analyzed and interpreted the patient data, and were major contributors in the development of the first draft of the present manuscript. JZ and WT participated in the experimental design, tissue collection and experimental execution. $\mathrm{ZZ}$ participated in the experimental design and experimental execution, and reviewed and approved the final draft of the manuscript prior to submission.

\section{Ethics approval and consent to participate}

Ethics approval for the present study was provided by The First Affiliated Hospital of The Jinzhou Medical University Ethics Committee, and informed consent was obtained from each participant prior to enrollment.

\section{Patient consent for publication}

Not applicable.

\section{Competing interests}

The authors declare that they have no competing interests.

\section{References}

1. Kadow T, Sowa G, Vo N and Kang JD: Molecular basis of intervertebral disc degeneration and herniations: What are the important translational questions? Clin Orthop Relat Res 473: 1903-1912, 2015.

2. Andersson GB: Epidemiological features of chronic low-back pain. Lancet 354: 581-585, 1999.

3. Chen Y, Wu Y, Shi H, Wang J, Zheng Z, Chen J, Chen X, Zhang Z, Xu D, Wang X and Xiao J: Melatonin ameliorates intervertebral disc degeneration via the potential mechanisms of mitophagy induction and apoptosis inhibition. J Cell Mol Med 23: 2136-2148, 2019.

4. Vergroesen PP, Kingma I, Emanuel KS, Hoogendoorn RJ, Welting TJ, van Royen BJ, van Dieën JH and Smit TH: Mechanics and biology in intervertebral disc degeneration: A vicious circle. Osteoarthritis Cartilage 23: 1057-1070, 2015.

5. Cheung KM, Karppinen J, Chan D, Ho DW, Song YQ, Sham P, Cheah KS, Leong JC and Luk KD: Prevalence and pattern of lumbar magnetic resonance imaging changes in a population study of one thousand forty-three individuals. Spine (Phila Pa 1976) 34: 934-940, 2009.

6. Yorimitsu E, Chiba K, Toyama Y and Hirabayashi K: Long-term outcomes of standard discectomy for lumbar disc herniation: A follow-up study of more than 10 years. Spine (Phila Pa 1976) 26: 652-657, 2001

7. Wang Y, Jiang L, Dai G, Li S and Mu X: Bioinformatics analysis reveals different gene expression patterns in the annulus fibrosis and nucleus pulpous during intervertebral disc degeneration. Exp Ther Med 16: 5031-5040, 2018.

8. Takashima Y, Kawaguchi A, Iwadate Y, Hondoh H, Fukai J, Kajiwara K, Hayano A and Yamanaka R: MicroRNA signature constituted of miR-30d, miR-93, and miR-181b is a promising prognostic marker in primary central nervous system lymphoma. PLoS One 14: e210400, 2019. 
9. Gagliardi D, Comi GP, Bresolin N and Corti S: MicroRNAs as regulators of cell death mechanisms in amyotrophic lateral sclerosis. J Cell Mol Med 23: 1647-1656, 2019.

10. Zhang P, Li S, Lv C, Si J, Xiong Y, Ding L, Ma Y and Yang Y: BPI-9016M, a c-Met inhibitor, suppresses tumor cell growth, migration and invasion of lung adenocarcinoma via miR203-DKK1. Theranostics 8: 5890-5902, 2018.

11. Li M, Ding W, Tariq MA, Chang W, Zhang X, Xu W, Hou L, Wang Y and Wang J: A circular transcript of ncx 1 gene mediates ischemic myocardial injury by targeting miR-133a-3p. Theranostics 8: 5855-5869, 2018.

12. Yang L, Cai Y, Zhang D, Sun J, Xu C, Zhao W, Jiang W and Pan C: miR-195/miR-497 regulate CD274 expression of immune regulatory ligands in triple-negative breast cancer. J Breast Cancer 21: 371-381, 2018.

13. Kong L, Sun M, Jiang Z, Li L and Lu B: MicroRNA-194 inhibits lipopolysaccharide-induced inflammatory response in nucleus pulposus cells of the intervertebral disc by targeting TNF receptor-associated factor 6 (TRAF6). Med Sci Monu 24: 3056-3067, 2018

14. Zhang B, Guo W, Sun C, Duan HQ, Yu BB, Mu K, Guan YY, Li Y, Liu S, Liu Y, et al: Dysregulated MiR-3150a-3p promotes lumbar intervertebral disc degeneration by targeting aggrecan. Cell Physiol Biochem 45: 2506-2515, 2018

15. Meng X, Zhu Y, Tao L, Zhao S and Qiu S: MicroRNA-125b-1-3p mediates intervertebral disc degeneration in rats by targeting teashirt zinc finger homeobox 3. Exp Ther Med 15: 2627-2633, 2018.

16. Li Z, Tao Y, Wang X, Jiang P, Li J, Peng M, Zhang X, Chen K, Liu H, Zhen P, et al: Tumor-secreted exosomal miR-222 promotes tumor progression via regulating P27 expression and Re-localization in pancreatic cancer. Cell Physiol Biochem 51: 610-629, 2018

17. Zong Y, Zhang Y, Sun X, Xu T, Cheng X and Qin Y: miR-221/222 promote tumor growth and suppress apoptosis by targeting lncRNA GAS5 in breast cancer. Biosci Rep 39: pii: BSR20181859, 2019.

18. Zhu Z, Zhang D, Lee H, Menon AA, Wu J, Hu K and Jin Y: Macrophage-derived apoptotic bodies promote the proliferation of the recipient cells via shuttling microRNA-221/222. J Leukoc Biol 101: 1349-1359, 2017.

19. Moses BS, Evans R, Slone WL, Piktel D, Martinez I, Craig MD and Gibson LF: Bone marrow microenvironment niche regulates miR-221/222 in acute lymphoblastic leukemia. Mol Cancer Res 14: 909-919, 2016.

20. Abo ElAtta AS, Ali YBM, Bassyouni IH and Talaat RM Upregulation of miR-221/222 expression in rheumatoid arthritis (RA) patients: Correlation with disease activity. Clin Exp Med 19: 47-53, 2019.
21. Livak KJ and Schmittgen TD: Analysis of relative gene expression data using real-time quantitative PCR and the 2(-Delta Delta C(T)) method. Methods 25: 402-408, 2001.

22. Nie X and Tian H: Correlation between miR-222 and uterine cancer and its prognostic value. Oncol Lett 16: 1722-1726, 2018.

23. Elmore S: Apoptosis: A review of programmed cell death. Toxicol Pathol 35: 495-516, 2007.

24. Yu W, Fu J, Liu Y, Wu Y and Jiang D: Osteogenic protein-1 inhibits nucleus pulposus cell apoptosis through regulating the $\mathrm{NF}-\kappa \mathrm{B} / \mathrm{ROS}$ pathway in an inflammation environment. Biosci Rep 38: pii: BSR20181530, 2018.

25. Xu Y, Yao H, Wang Q, Xu W, Liu K, Zhang J, Zhao H and Hou G: Aquaporin-3 attenuates oxidative stress-induced nucleus pulposus cell apoptosis through regulating the P38 MAPK pathway. Cell Physiol Biochem 50: 1687-1697, 2018.

26. Zhang CZ, Kang CS, Pu PY, Wang GX, Jia ZF, Zhang AL, Han L and Xu P: Inhibitory effect of knocking down microRNA-221 and microRNA-222 on glioma cell growth in vitro and in vivo. Zhonghua Zhong Liu Za Zhi 31: 721-726, 2009 (In Chinese).

27. Nguyen KC, Willmore WG and Tayabali AF: Cadmium telluride quantum dots cause oxidative stress leading to extrinsic and intrinsic apoptosis in hepatocellular carcinoma HepG2 cells. Toxicology 306: 114-123, 2013.

28. Li X, Fang F, Gao Y, Tang G, Xu W, Wang Y, Kong R, Tuyihong A and Wang Z: ROS induced by KillerRed targeting mitochondria (mtKR) enhances apoptosis caused by radiation via Cyt c/caspase-3 pathway. Oxid Med Cell Longev 2019: 4528616, 2019.

29. Eskes R, Antonsson B, Osen-Sand A, Montessuit S, Richter C, Sadoul R, Mazzei G, Nichols A and Martinou JC: Bax-induced cytochrome $\mathrm{C}$ release from mitochondria is independent of the permeability transition pore but highly dependent on $\mathrm{Mg} 2+$ ions J Cell Biol 143: 217-224, 1998.

30. Sinha K, Das J, Pal PB and Sil PC: Oxidative stress: The mitochondria-dependent and mitochondria-independent pathways of apoptosis. Arch Toxicol 87: 1157-1180, 2013.

31. Miyazaki S, Kakutani K, Yurube T, Maeno K, Takada T,Zhang Z, Kurakawa T, Terashima Y, Ito M, Ueha T, et al: Recombinant human SIRT1 protects against nutrient deprivation-induced mitochondrial apoptosis through autophagy induction in human intervertebral disc nucleus pulposus cells. Arthritis Res Ther 17: $253,2015$.

This work is licensed under a Creative Commons Attribution-NonCommercial-NoDerivatives 4.0 International (CC BY-NC-ND 4.0) License. 